\title{
2D Phase Purity Determines Charge Transfer Yield at 3D/2D Lead Halide Perovskite Heterojunctions
}

Robert J. E. Westbrook ${ }^{1,2,3 *}$, Weidong $\mathrm{Xu}^{1,3}$, Xinxing Liang ${ }^{1,3}$, Thomas Webb, ${ }^{4}$ Tracey M. Clarke ${ }^{2}$, Saif A. Haque ${ }^{1,3 *}$

${ }^{1}$ Department of Chemistry, Imperial College London, Molecular Sciences Research Hub White City Campus, Wood Lane, W12 OBZ, UK

${ }^{2}$ Department of Chemistry, University College London, 20 Gordon Street, London WC1H OAJ, UK ${ }^{3}$ Centre for Processable Electronics, Imperial College London, London SW7 2AZ, UK

${ }^{4}$ Advanced Technology Institute, Department of Electrical and Electronic Engineering, University of Surrey, Guildford, Surrey, GU2 7XH, UK

*E-mail: R.J.E.W (r.westbrook15@imperial.ac.uk); S.A.H (s.a.haque@imperial.ac.uk) Supporting Information: 


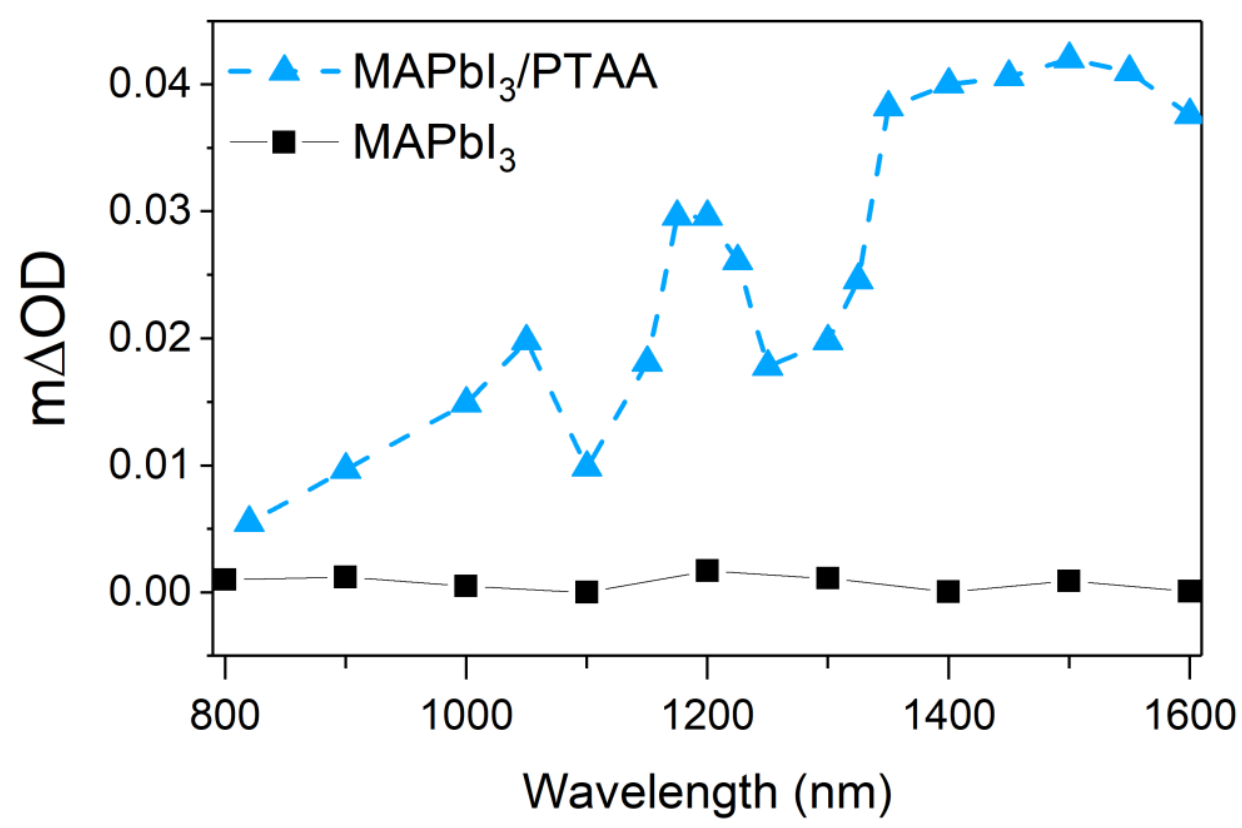

Figure S1: Transient absorption spectrum of $\mathrm{MAPbl}_{3}$ and $\mathrm{MAPbl}_{3} \mid \mathrm{PTAA}$ captured $10^{-6} \mathrm{~s}$ after excitation at $510 \mathrm{~nm}$ (fluence: $10 \mu \mathrm{J} \mathrm{cm}^{-2}$ )

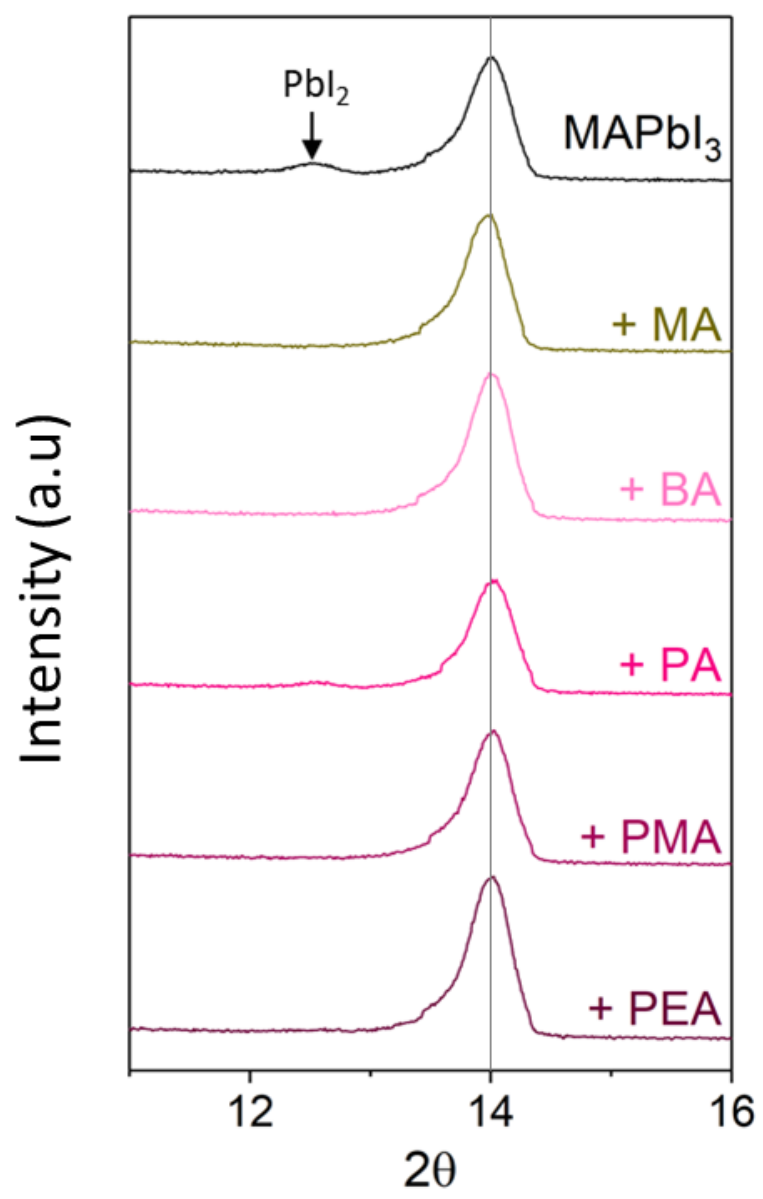

Figure S2: Grazing incidence X-ray diffraction (GIXRD) patterns for a $\mathrm{MAPb}_{3}$ film without treatment and passivated with $10 \mathrm{mM}$ MAI, BAI, PAI, PMAl and PEAI. 


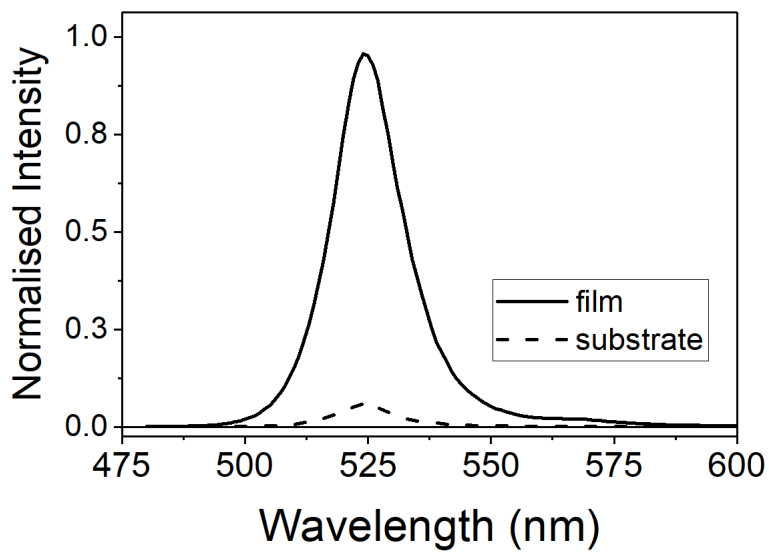

Figure S3: Steady-state photoluminescence spectra of 3D/RDP, formed after treatment of $\mathrm{MAPbl}_{3}$ with $20 \mathrm{mM}$ PEAl, measured with excitation $(450 \mathrm{~nm}$ ) at the film (solid line) and substrate (dashed line) faces.

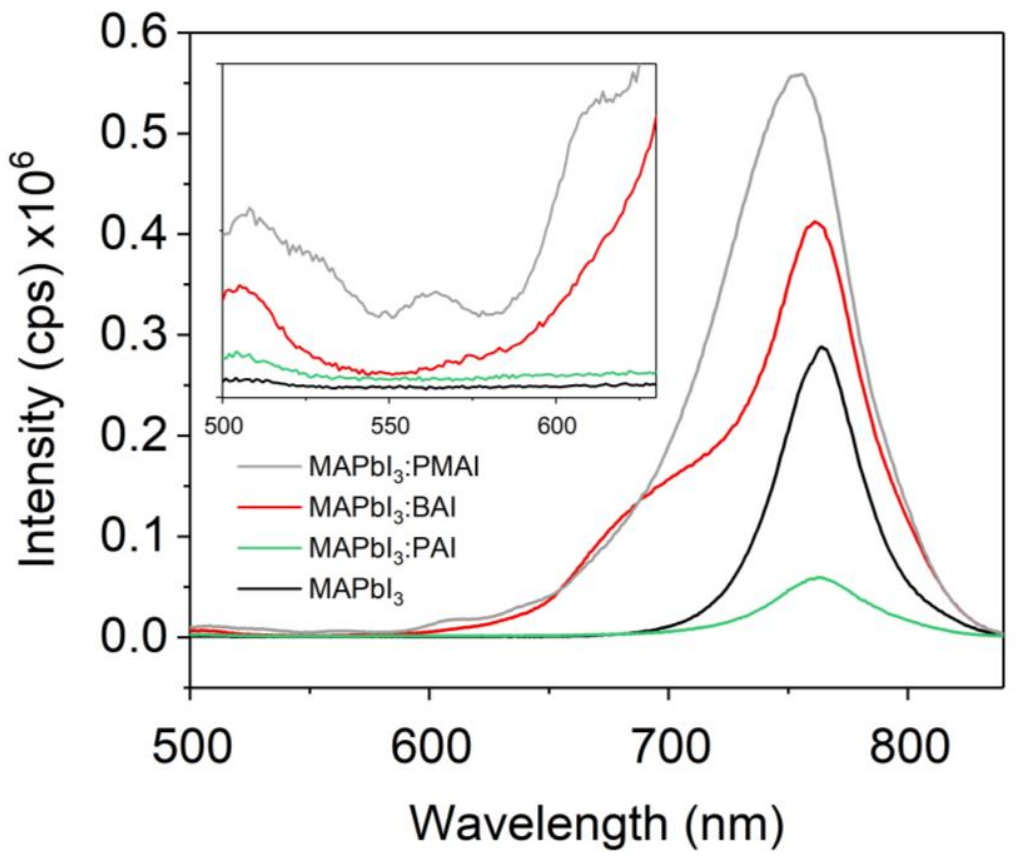

Figure S4: Photoluminescence spectra of $\mathrm{MAPbl}_{3}$ films treated with $10 \mathrm{mM} \mathrm{PMAl}$ (grey), BAI (red), PAI (green) and with no treatment (black). Samples were excited at $435 \mathrm{~nm}$ via the film surface. 


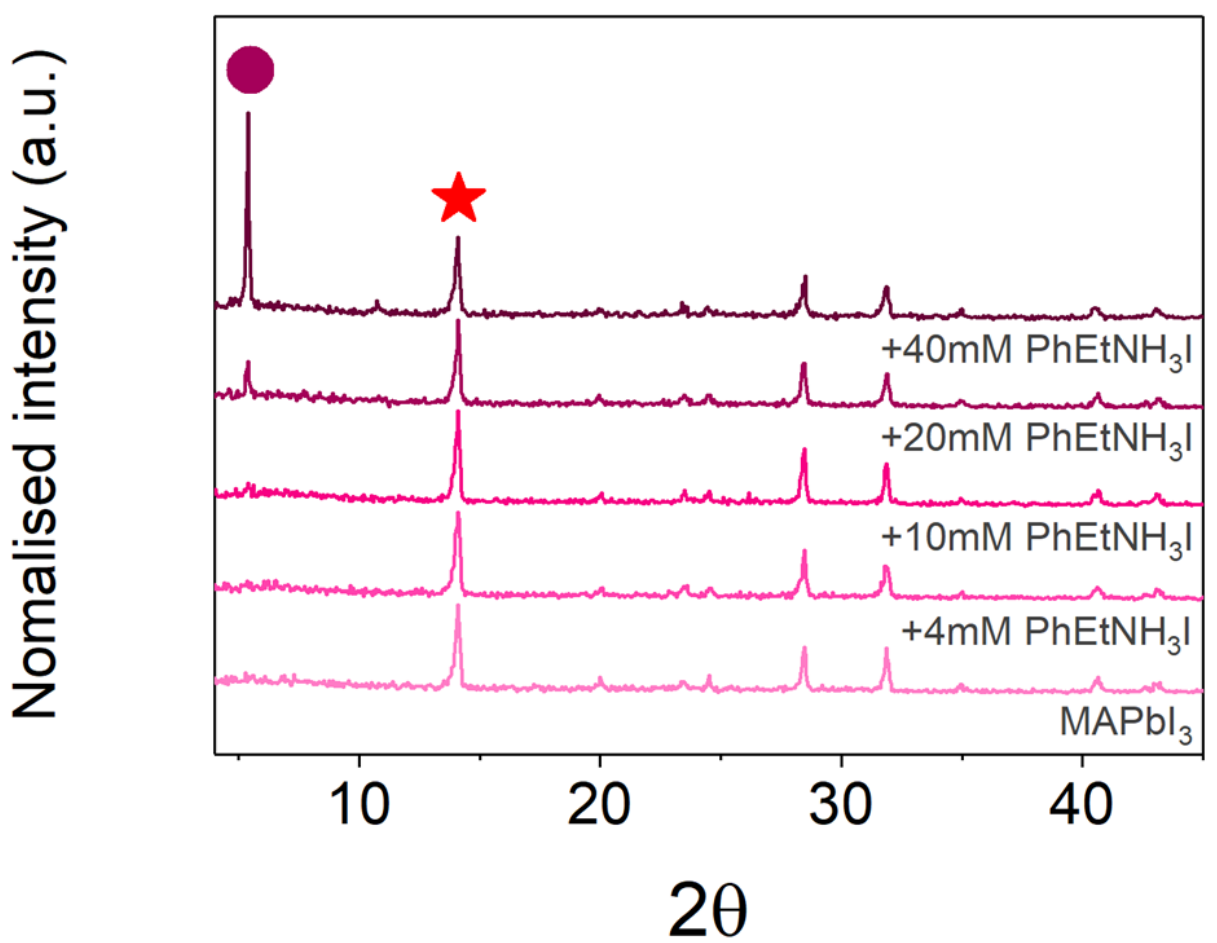

Figure S5: X-ray diffraction patterns for $\mathrm{MAPbl}_{3}$ films treated with 0, 4, 10, 20 and $40 \mathrm{mM}$ PEAI. The

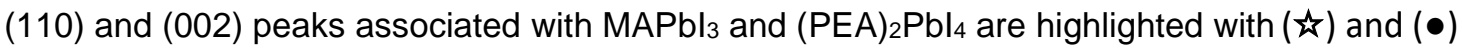
respectively.

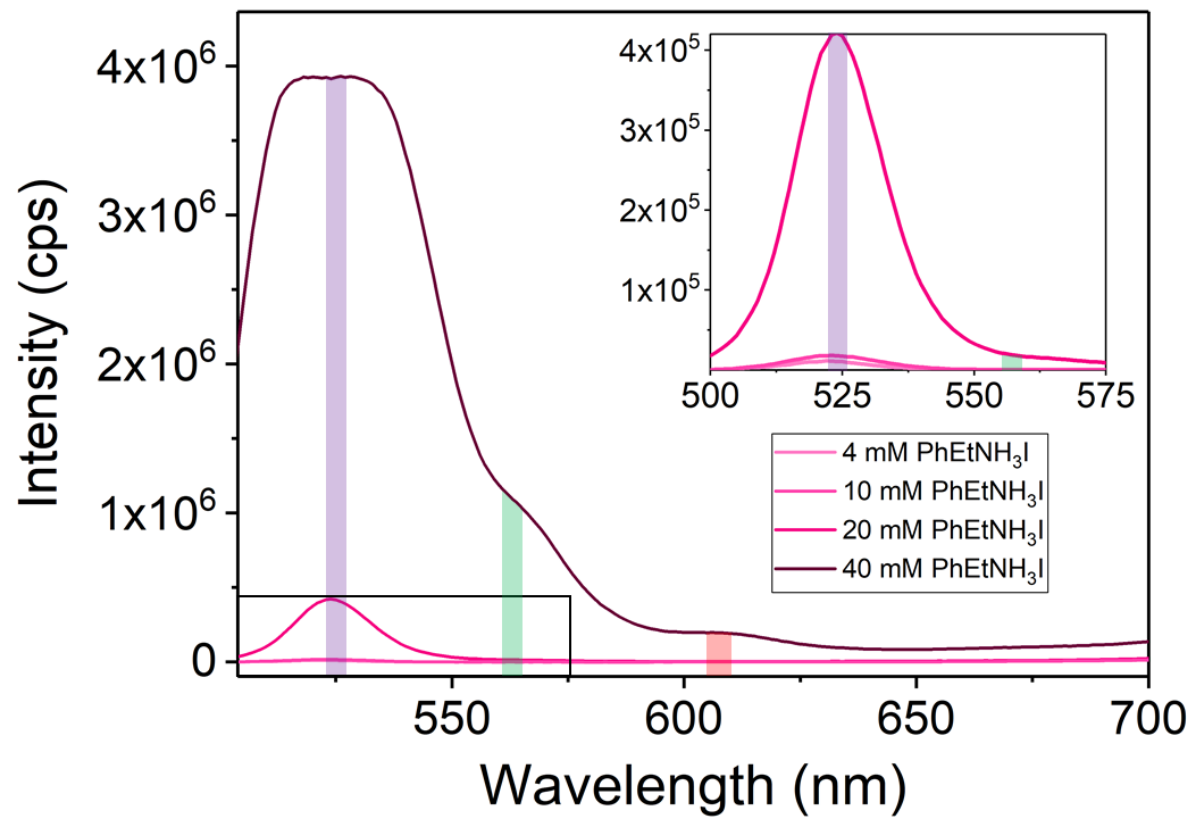

Figure S6: Raw photoluminescence spectra of $\mathrm{MAPbl}_{3}$ treated with 4, 10, 20 and $40 \mathrm{mM}$ of PEAl. Increasing concentration is represented by darker shades. Samples were excited at $435 \mathrm{~nm}$ via the film surface. Contributions from $\left.(P E A)_{2}(M A)_{n-1} P_{n}\right|_{3 n+1}$ are highlighted for the case where $n=1$ (purple); 2 (green); and 3 (red). Inset shows a zoom of the PL spectra indicated in the box. We note that there is evidence of detector saturation at $525 \mathrm{~nm}$ for the $40 \mathrm{mM}$ sample, leading to an underestimate of the $n=1$ contribution in this case. 




Figure S7: Absorption spectra of 3D, 2D and 3D/RDP formed after treatment of $\mathrm{MAPbl}_{3}$ with $10 \mathrm{mM}$ PEAI.
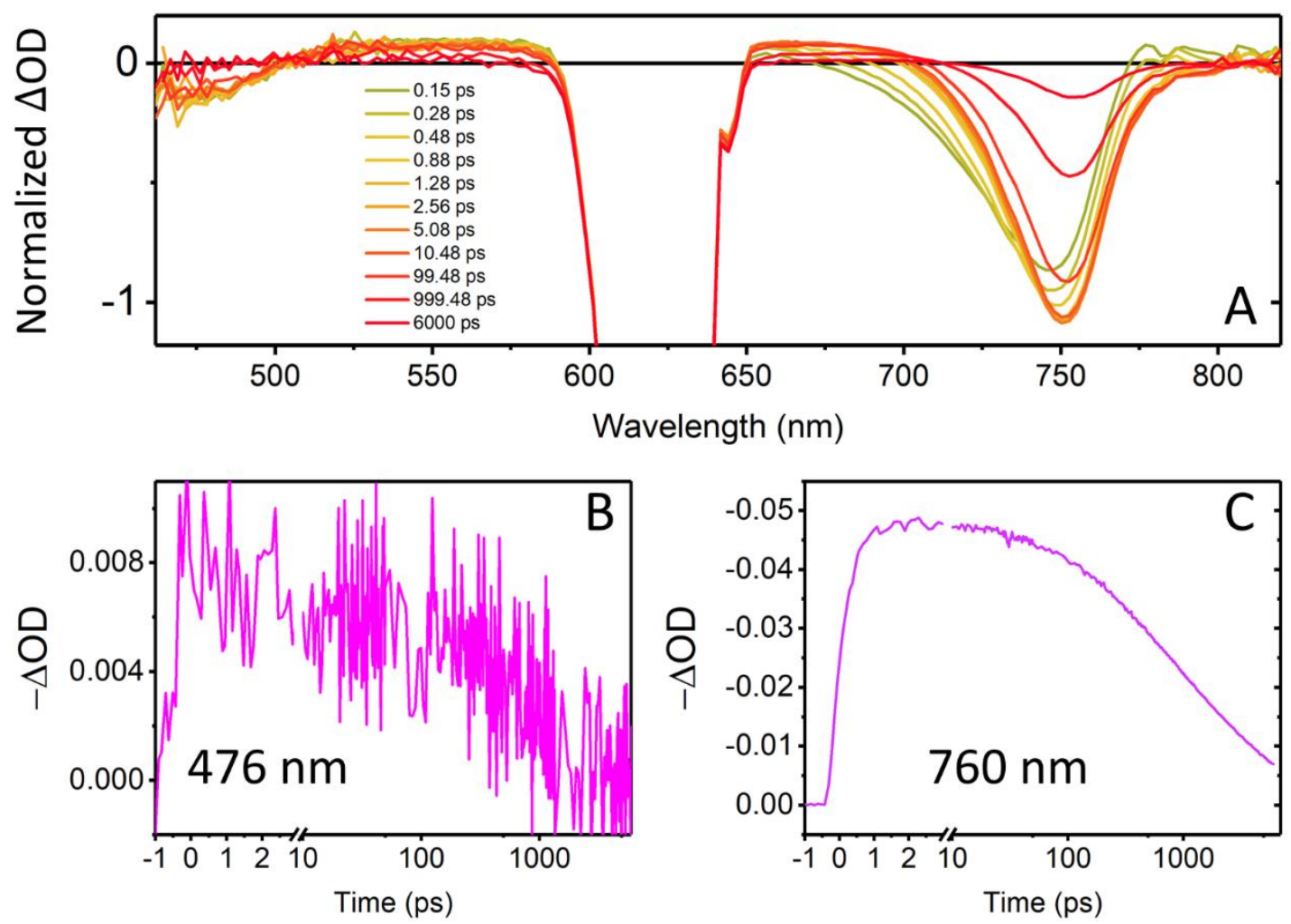

Figure S8: (a) Femtosecond transient absorption spectra as a function of time for a 3D/RDP film formed after treatment of $\mathrm{MAPbl}_{3}$ with $40 \mathrm{mM}$ PEAl. The decay dynamics at $476 \mathrm{~nm}$ and $760 \mathrm{~nm}$ are shown in (b) and (c) respectively. The films were pumped at $635 \mathrm{~nm}$ (fluence: $25 \mu \mathrm{J} \mathrm{cm}^{-2}$ ). The contribution between $600-650 \mathrm{~nm}$ is associated with the pump pulse. 


\section{Materials \& Methods}

\section{Sample Preparation}

Glass slides were cut to $1 \times 1 \mathrm{~cm}$ and washed in detergent, acetone, water and isopropanol sequentially. Mesoporous titanium dioxide $\left(\mathrm{mp}-\mathrm{TiO}_{2}\right)$ substrates were prepared by spin-coating commercial $\mathrm{mp}-\mathrm{TiO}_{2}$ paste (Dyesol), diluted in ethanol 2:7 (paste: ethanol), at $6000 \mathrm{rpm}$ for $30 \mathrm{~s}$ onto the clean glass slides. They were then annealed at $500{ }^{\circ} \mathrm{C}$ for 45 minutes. The $\mathrm{MAPbl}_{3}$ deposition method was adapted from that of Jeon et al. ${ }^{38}$ The $\mathrm{MAPbl}_{3}$ precursor solution contained a 1:1 molar ratio of $\mathrm{Pbl}_{2}$ (Alpha Aesar) and MAI (Dyesol) at a concentration of $1.2 \mathrm{M}$ in a mixture of $\mathrm{Y}^{-}$ Butyrolactone and dimethyl sulfoxide (ratio 3:7). Once properly mixed, the resulting solution was spin-coated onto the relevant substrate in a 3-step program at 1000, 5000 and $6000 \mathrm{rpm}$ for 10,30 and $20 \mathrm{~s}$ respectively. $800 \mu \mathrm{L}$ of toluene was drop casted onto the spinning film between the second and third step to aid crystallisation. After the spin-coating program, the film was annealed on a 100 ${ }^{\circ} \mathrm{C}$ hotplate for 10 minutes. The formation of the perovskite was indicated by a colour change from pale yellow to dark brown.

Once cooled, iodide salt coatings were added via the method first reported by Aristidou et al. ${ }^{8}$ Briefly, solutions were prepared by dissolving the desired concentration of iodide salt (ammonium iodide, methylammonium iodide, n-butylammonium iodide, phenylammonium iodide, phenylmethylammonium iodide, phenylethylammonium iodide) in a 1:4 solvent mixture of IPA and chlorobenzene (CB). All salts were acquired from Sigma Aldrich except methylammonium iodide, which was from Dyesol. One hundred microlitres of salt solution was dripped onto pre-deposited perovskite films with a $20 \mathrm{~s}$ loading time before spinning at $4,000 \mathrm{rpm}$ and annealing at $100{ }^{\circ} \mathrm{C}$ for 5 min. This was followed by a washing step, in which the film was treated with one hundred microlitres of the 1:4 IPA:CB mixture and spin-coated at 4,000 rpm. HTLs, if relevant, were subsequently deposited via spin-coating such that a $30 \pm 5 \mathrm{~nm}$ thick layer was formed. PTAA $\left(M_{w}=\right.$ $28.422 \mathrm{kDa}$ ) and PTPD ( $\mathrm{M}_{\mathrm{w}}=80,000 \mathrm{kDa}$ ) were deposited from $10 \mathrm{mg} / \mathrm{mL}$ and $8 \mathrm{mg} / \mathrm{mL}$ solutions respectively, in CB and spin-coated at 5000 and 2500 rpm respectively. 


\section{Transient Absorption Spectroscopy:}

TAS is a pump-probe technique that operates on the idea that photogenerated charges change the optical density (OD) property of semiconductors. An initial 'pump' laser pulse, with energy larger than the band-gap, is used to excite electrons from the ground $\left(\psi_{0}\right)$ to an excited state $\left(\psi_{1}\right)$. Subsequently, a second beam probes the absorbance of $\psi_{1}$ by exciting it to $\psi_{1}$ (Figure S9). In the case of our measurements on 3D/RDP|HTL films, $\psi_{1}$ is the HTL after hole injection i.e. the $\mathrm{HTL}^{+}$ polaron. Importantly, the initial change in optical density, $\triangle O D_{\max }$ at the wavelength characteristic of the oxidised HTL is proportional to the concentration, $n_{1}$ of $\psi_{1}$ by the Beer-Lambert law $-\triangle O D=$ $\varepsilon_{1} n_{1} l$, where $\varepsilon_{1}$ and $n_{1}$ are the extinction coefficient of the excited state and path length respectively. Therefore, $\triangle O D_{\max }$ is a direct indicator of the hole injection yield to the HTL.

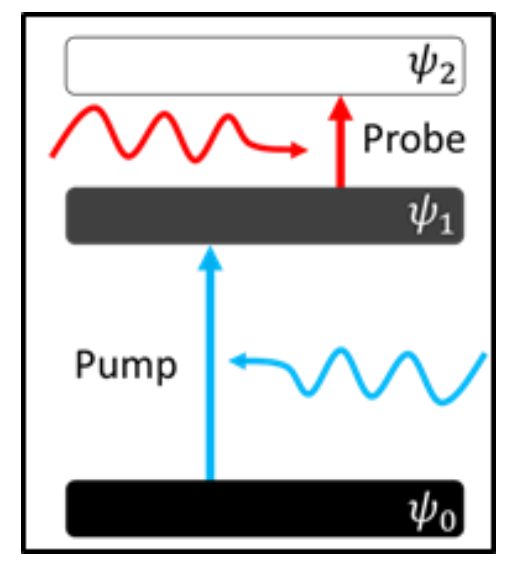

Figure S9: Three state diagram for transient absorption spectroscopy.

Transient absorption spectroscopy of $\mathrm{MAPbl}_{3}$ films was done with a $510 \mathrm{~nm}$ excitation at an initial fluence of $10 \mu \mathrm{Jm}^{-2}$. A probe wavelength characteristic of the hole or electron polaron in the CTL was used to monitor charge injection (1600 nm for PTPD; 1500 for PTAA). Briefly, we held our sample films in a sealed cuvette under a steady purge of $\mathrm{N}_{2}$. We then exposed the sample to the pulsed $(3 \mathrm{~Hz}$ ) pump and continuous probe beams. The probe beam was generated by a $100 \mathrm{~W}$ tungsten lamp (Bentham IL 1) and filtered by a monochromator (OBB-2001, Photon technology international) and a long-pass filter before it was collimated and focused on the sample. On a 
separate optical axis, the pump beam was generated by a tuneable dye laser (Photon Technology International Int. GL-301; pulse width $1.6 \mathrm{~ns}$ ) that was itself excited by a $\mathrm{N}_{2}$ laser (Photon Technology International Inc. GL-3300). We controlled the laser fluence (maximum: $10 \mu \mathrm{J} \mathrm{cm}^{-2}$ ) by inserting ND filters between the laser head and the sample. The transient changes in the absorption of the sample were detected by a silicon photodiode (Hamatsu Photonics, S1722-01) after filtering with more long-pass filters in order to remove the contribution from the sample PL. The signal was then amplified (Costronics Electronics) and finally interpreted by a digital oscilloscope (Tektronics DPO3012).

Ultrafast transient absorption spectroscopy measurements were conducted by using a transient absorption spectrometer (HELIOS) with a $635 \mathrm{~nm}$ pulsed excitation laser pump and a pulsed white light probe. The pump was generated via an optical parametric amplifier (TOPAS Prime, SpectraPhysics) while the probe was generated through a sapphire crystal. Both pump and probe have a frequency of $500 \mathrm{~Hz}$ and are original from Solstice Ti:Sapphire regenerative amplifier (Newport, Spectra-Physics) with an instrument response time of $200 \mathrm{fs}$. The intensity of pump was measured of $24 \mu \mathrm{J} / \mathrm{cm}^{2}$ per pulse by using VEGA power meter from OPHIR Photonics.

Photoluminescence and Absorbance Spectroscopy: Steady-state photoluminescence was carried out with a commercial Horiba Fluorolog 1039 spectrometer (excitation wavelength: 510 nm; probe: $760 \mathrm{~nm}$ ). Time-correlated single photon counting (TCSPC) was carried out with the same probe wavelength at an excitation of $635 \mathrm{~nm}\left(0.11 \mathrm{~nJ} \mathrm{~cm}^{-2}\right)$, with a commercial Horiba Deltaflex system. The excitation source for the TCSPC was a Horiba NanoLED-635L with a fluence of $0.11 \mathrm{~nJ}$ $\mathrm{cm}^{-2}$, repetition rate of $1 \mathrm{MHz}$ and pulse half-life of $120 \mathrm{ps}$. UV-Vis spectra were captured with a Shimadzu UV-2600 integrating sphere spectrophotometer.

The excitation source for the TCSPC was a Horiba nanoLED-370 with an excitation wavelength of 369 $\mathrm{nm}$, a pulse duration of $1.3 \mathrm{~ns}$, and a repetition rate of $1 \mathrm{MHz}$ 
X-Ray Diffraction: XRD patterns were recorded using a Bruker D8 Discover X-ray diffractometer using monochromatic $\mathrm{Cu} \mathrm{K}_{\alpha 1}$ and $\mathrm{Cu} \mathrm{K}_{\alpha 2}$ radiation of wavelengths 1.54056 and $1.54439 \AA$, respectively. The Grazing Incidence X-ray diffraction (GIXRD) crystallographic data for the samples was collected using a PANalytical XPert PRO diffractometer using a GI thin film bracket stage and monochromatic $\mathrm{Cu} \mathrm{K} \mathrm{K}_{\alpha 1}$ and $\mathrm{Cu} \mathrm{K}_{\alpha 2}$ radiation of wavelengths 1.54056 and $1.54439 \AA$ ( $40 \mathrm{~mA}, 45 \mathrm{kV}$ ). 